\title{
Ocorrência de pneumonia aspirativa em crianças disfágicas pós videofluoroscopia
}

\section{Occurrence of aspiration pneumonia in dysphagic children post video fluoroscopy}

\author{
Hellen Nataly Correia Lagos', Rosane Sampaio Santos ${ }^{2}$, Adriane Celli ${ }^{3}$, Edna Marcia Silva Abdulmassih', \\ Carlos Alberto do Amaral Medeiros4.
}

1) Graduação. Fonoaudióloga.

2) Mestrado. Fonoaudióloga.

3) Doutorado. Gastropediatra

4) Residência. Residente em Pediatria.

Instituição: Universidade Tuiuti do Paraná. Curitiba / PR - Brasil.

Endereço para correspondência: Hellen Nataly Correia Lagos - Rua Santa Catarina, 427 - Apto 24 - Bairro: Água Verde - Curitiba / PR - Brasil - CEP: 80 620-100 - Telefone: (+55 41) 8416-2860 - E-mail: hellennataly@ gmail.com

Fonte financiadora ou fornecedora dos equipamentos: Hospital de Clínicas da Universidade Federal do Paraná

Artigo recebido em 15 de Março de 2011. Artigo aprovado em 25 de Junho de 2011

RESUMO

Introdução: A literatura relata que quando se trata de avaliação instrumental da deglutição em crianças, sem dúvida, a videofluoroscopia da deglutição oferece grandes vantagens sobre o estudo endoscópico (6).

Objetivo: Verificar o risco de pneumonia aspirativa após a realização do estudo da deglutição por vídeofluoroscopia, em crianças com disfagia.

Método: Em estudo de corte prospectivo, participaram 16 crianças com idade entre 6 meses e 10 anos, com média de 5,2 anos, encaminhadas para estudo da deglutição por videofluoroscopia. Foram testadas 4 consistências, pudim, néctar, mel e líquido. A presença de sinais e/ou sintomas respiratórios foram avaliados pré e pós estudo da deglutição por videofluoroscopia, por meio de histórico e exame clínico. Quando necessário solicitado radiografia de tórax.

Resultados: Das 16 crianças, 5 não apresentaram disfagia. Em 11 crianças o exame demonstrou 4 com disfagia leve, 2 moderada e 5 grave, conforme Classificação de OTT (1996) Classificação da gravidade da disfagia à videofluoroscopia9. Das 7 crianças que aspiraram durante a realização do exame, apenas 1 apresentou sintomas respiratórios após o estudo da deglutição, porém sem sinais de pneumonia ao exame físico. Conclusão: $\mathrm{Na}$ população estudada, não houve ocorrência de pneumonia aspirativa após a realização do estudo da deglutição por vídeofluoroscopia, apesar da ocorrência de aspiração durante o exame em cerca de 50\% dos casos.

Palavras-chaves: transtorno de deglutição, fluoroscopia, pneumonia aspirativa.

\section{SUMMARY}

Introduction: The literature reports that when it comes of instrumental assessment of swallowing in children, undoubtedly, video fluoroscopy of swallow offers great advantages over the endoscopic study.

Objective: Check the risk of aspiration pneumonia after the study of swallowing by video fluoroscopy, in children with dysphagia.

Method: In a study of prospective cutting, participated 16 children aged between 6 months and 10 years, with an average of 5,2 years, referred for study of swallowing by video fluoroscopy. Were tested 4 consistencies, pudding, nectar, honey and liquid. The presences of signs and/or respiratory symptoms were evaluated pre and post study of deglutition by video fluoroscopy, through history and clinical exam. When necessary was asked chest $\mathrm{x}$-ray.

Results: Of 16 children, 5 didn't presented dysphagia. In 11 children the exam showed 4 with mild dysphagia, 2 moderate and 5 severe, as classification of OTT (1996) - Classification of severity of dysphagia to the video fluoroscopy. Of the 7 children who aspirated during the exam, only 1 presented respiratory symptoms after the deglutition study, but without signal of pneumonia to the physical examination.

Conclusion: In the studied population there were no occurrences of aspiration pneumonia after the study of deglutition was performed by video fluoroscopy, despite the occurrence of aspiration during the exam in about $50 \%$ of cases.

Keywords: deglutition disorders, aspiration pneumonia, fluoroscopy. 


\section{INTRODUÇÃO}

Nas disfagias orofaringeanas, as complicações mais difíceis de gerenciamento clínico são as afecções pulmonares causadas pela aspiração. Assim, a detectabilidade e caracterização dessa aspiração, que ocorre na fase faríngea, são primordiais para o prognóstico e reabilitação. Pode-se inferir a aspiração pela avaliação clínica, mas sua comprovação objetiva deve ser realizada com a videofluoroscopia (1).

A videofluoroscopia da deglutição é considerado o melhor método instrumental de avaliação da deglutição em crianças, pois avalia desde o início da fase preparatória oral até a conclusão da fase esofágica (2). Porém, trabalhos apontam que crianças com disfagia grave possuem alto risco de complicações durante o exame (3).

O exame instrumental como a videofluoroscopia é um grande aliado na avaliação de bebês e crianças com distúrbios da deglutição. Com este exame tem se observado a alta incidência de aspiração silenciosa em estudos radiográficos envolvendo crianças com múltiplas deficiências (1).

Os pacientes com disfagia têm risco aumentado de aspiração, ou seja, da passagem de alimentos e/ou fluídos abaixo das pregas vocais entrando nas vias respiratórias, aumentando o risco de pneumonia e problemas associados (4). Ainda segundo estes autores, a maioria dos estudos sobre a aspiração silenciosa em paciente disfágico, defende o uso da videofluoroscopia da deglutição para sua detecção, considerado o exame mais sensível em detecção de aspiração durante a deglutição (2).

Para Langmore (1999) são necessários três prérequisitos para o desenvolvimento da pneumonia aspirativa: a) germe patogênico presente no material a ser aspirado; b) o material precisa ser aspirado e c) os pulmões precisam ser incapazes de resistir aos germes patogênicos $(5,6)$.

$\mathrm{Na}$ criança, sem dúvida alguma, a videofluoroscopia da deglutição oferece grandes vantagens sobre o estudo endoscópico, além de ser tecnicamente mais factível, reproduz praticamente a situação real da deglutição (7).

Apesar das vantagens, como a análise dinâmica precisa e imediata da deglutição e o procedimento não invasivo, há para a população pediátrica um risco maior que no adulto, pois a radiossensibilidade da tiroide é conhecida por ser particularmente elevada (8).

Durante a realização do exame, o examinador deve observar se há ou não sinais de percepção da aspiração laringotraqueal, como tossir, engasgar, limpar a garganta e lacrimejar. Se o paciente não mostrar nenhum desses sinais, será considerado um aspirador silencioso, com risco de desenvolver pneumonia por aspiração (9). Alguns autores referem que se houver aspiração significativa do bolo sem limpeza reflexa ou voluntária das vias aéreas, o exame deverá ser encerrado, pois embora o bário seja considerado relativamente inerte, a entrada de uma grande quantidade na árvore respiratória não é recomendável, uma vez que pode causar problemas a pessoas com doenças sistêmicas ou respiratórias.

Este trabalho tem como objetivo verificar a ocorrência de pneumonia aspirativa após a realização do exame de videofluoroscopia, em crianças de alto risco, por meio de acompanhamento fonoaudiológico e médico, após a realização do mesmo.

\section{MÉTODO}

Tratou-se de um estudo de corte prospectivo em 16 crianças com idade entre 6 meses e 10 anos, com média de 5,2 anos, que haviam sido encaminhadas pelo departamento de pediatria para estudo da deglutição por videofluoroscopia para investigação de disfagia. Sendo $50 \%$ do sexo masculino e $50 \%$ do sexo feminino, com peso médio de $14 \mathrm{~kg}$, com queixa de disfagia.

Na descrição da doença de base, 13 crianças apresentavam Paralisia Cerebral, $1 \mathrm{com}$ Microcefalia, $2 \mathrm{com}$ Encefalopatia .

Passaram pelo estudo todas as crianças com sintomas de disfagia que foram encaminhadas para realizar o estudo da deglutição por videofluoroscopia.

Os critérios para inclusão das crianças na pesquisa foram: condição clínica para a realização do exame, estar acordada, ativa e com liberação médica. Foram excluídas da pesquisa todas aquelas que estavam com quadro de pneumonia antes da realização do exame.

O estudo foi aprovado pelo Comitê de Ética em Pesquisa, sob o no. 000025/2009, (Anexo I).

Para coleta de dados, sobre a doença de base, causa da disfagia e sinais e sintomas de pneumonia, foram utilizados os prontuários dos pacientes e entrevista inicial com o responsável pela criança, e os mesmos assinaram um termo autorizando a utilização dos dados na pesquisa.

Foi utilizada a Functional Oral Intake Scale - FOIS, a qual segue uma escala de Nível 1 até o Nível 7 , sendo que quanto menor o valor menor é a ingestão de alimento via oral (10), para classificar a ingesta por via oral das crianças. 
Antes da realização do exame de videofluoroscopia as crianças foram submetidas à radiografia de tórax para excluir possibilidade de pneumonia prévia, realizado com o aparelho, marca Siemens e modelo Axiom R100, monitor Siemens e modelo M44-2, com o qual também foi realizado o exame da videofluoroscopia da deglutição.

Para a realização do exame as crianças eram posicionadas sentadas a $90^{\circ}$ sozinhas ou quando necessário no colo da mãe ou responsável, com visão radiografia lateral.

As consistências apresentadas foram Pudim, Mel, Néctar e Líquida, compostas porágua, Sulfato de bário 100\% da marca Bariogel, como contraste radiológico o qual contém sulfato de bário $1 \mathrm{~g}$ e veículo g.s.p. $1 \mathrm{ml}$, de uso pediátrico e adulto, e como espessante utilizamos o amido de milho modificado instantâneo da marca THICK UP ${ }^{\circledR}$ composto por amido de milho modificado (E1442), maltodextrina, goma de tara, goma de xantana e goma guar.

Para a obtenção das consistências, foi utilizada a nomenclatura da NationalDysphagia Diet: Standardization for Optimal Care (11) e o espessante THICK UP ${ }^{\circledR}$, onde foi utilizado para o néctar (de 51 a 350cP), -200 ml de água, 2 sachês (10 gramas) de THICK UP ${ }^{\circledR}$, Mel (de 351 a $1750 c P$ ) - 200ml de água, 21/2 sachês (12,5 gramas) de THICK UP ${ }^{\circledR}$ e Pudim(> que $1750 c P$ ), - 200ml de água, 3 sachês (15 gramas) de THICK UP ${ }^{\circledR}$. espessante alimentar instantâneo da marca Thick\&Easy ${ }^{\circledR}$ (HORMEL HEATH LABS. SWISS) composto de amido, apresentando como composição nutricional por 100g: 375kcal, $100 \mathrm{~g}$ de carboidratos e $125 \mathrm{mg}$ de sódio.

Os utensílios utilizados para oferecer as consistências, foram colher, copo e mamadeira no caso dos bebês.

Para gravação dos exames foi utilizado o computador de marca HP Pavilion tx2075BR Notebook PC e o coletor de imagem utilizado foi o Sapphire - Wonder TVUSB.

Para classificação do grau de disfagia, foi utilizada a Classificação da Gravidade da Disfagia à Videofluoroscopia
- OTT (1996), na qual se classifica Deglutição Normal, Disfagia Leve, Disfagia Moderada e Disfagia Grave (12).

Após a realização do exame os pacientes retornaram em no máximo uma semana para avaliação médica para verificar a existência de sinais ou sintomas respiratórios e avaliação clínica fonoaudiológica dos sinais e sintomas de disfagia, histórico de aspiração e condições clínicas por meio de um protocolo. Esse período de 7 dias para o retorno, foi em função da disponibilidade de retorno do público alvo, utilizando o limite de dias para tal avaliação. Foi realizada nova radiografia de tórax, quando os sinais e sintomas respiratórios eram sugestivos de pneumonia.

\section{RESULTADOS}

Dos encaminhamentos para a realização do estudo da deglutição por videofluoroscopia da deglutição, 6 crianças foram encaminhados por Gastroenterologista, 3 por Pediatra, 4 por Fonoaudiólogas, 2 por pneumologista e 1 por Imunologista.

Das 16 crianças apenas 2 utilizavam traqueostomia do tipo metal, e somente 1 encontrava-se internada.

Antes da realização do exame, 4 crianças apresentaram sintomas respiratórios, tosse com catarro, dificuldade respiratória, febre e tosse seca, porém sem diagnóstico de pneumonia.

Desses pacientes com sintomas respiratórios antes do exame 2 persistiram com os sintomas após a realização do exame e nas outras 2 crianças os sintomas desapareceram.

Com relação à ingesta por via oral pela Functional Oral Intake Scale - FOIS (9), 5 (31,25\%) encontravam-se no N1 (Nível 1), 1 (6,25\%) no N2, 1 (6,25\%) no N3, 6 (37,50\%) no N5 e 2 (12,50\%) no N7, já ocorrência de aspiração durante a videofluoroscopia foi em 7 (43,75\%) das crianças testadas, como mostra a Tabela 1.

Pela Classificação de OTT (11) da gravidade da disfagia à videofluoroscopia, $5(31,25 \%)$ crianças apresen-

Tabela I . Relação de ingesta por via oral -FOIS, aspiração traqueal durante a videofluoroscopia e classificação do grau de disfagia pela escala de OTT.

\begin{tabular}{lcccccccccccc}
\hline & \multicolumn{4}{c}{ Ingesta via oral - FOIS } & \multicolumn{4}{c}{$\begin{array}{c}\text { Aspiraçãotraqueal } \\
\text { no exame }\end{array}$} & \multicolumn{2}{c}{ Grau de disfagia pela classificação de OTT } \\
\hline N1 & N2 & N3 & N4 & N5 & N6 & N7 & P & A & Normal & Leve & Moderada & Grave \\
\hline 5 & I & I & 0 & 6 & 0 & 2 & $7(43,7)$ & $9(56,2)$ & $5(31,2)$ & $4(25,0)$ & $2(12,5)$ & $5(31,2)$ \\
\hline
\end{tabular}

Fonte: Aautora

Nota: $F O / S^{\otimes}$ escalafuncional de alimentação; $A=$ Ausente; $P=$ Presente; Número de pacientes(\%). 
taram deglutição normal, 4 (25\%) disfagia leve, 2 (12.50\%) disfagia moderada e $5(31,25 \%)$ disfagia grave (Tabela 1$)$.

Das $7(43,75 \%)$ crianças cujo exame demonstrou aspiração, 2 (28,5\%) aspiraram simultaneamente as consistências pudim, mel, néctar e líquido, outras 3 (42,8\%) crianças aspiraram mel, néctar e líquido e por fim, 2 $(28,5 \%)$ aspiraram somente a consistência líquida, como mostra a Tabela 2 .

Dentre as $7(43,75 \%)$ crianças, as quais apresentaram aspiração traqueal durante o exame, apenas $2(28,5 \%)$ apresentaram sintomas respiratórios após o estudo da deglutição, porém sem sinais de pneumonia ao exame físico (Tabela 3).

\section{DISCUSSÃO}

O presente estudo procurou verificar a ocorrência de pneumonia aspirativa após a realização do exame de videofluoroscopia da deglutição. Em revisão de literatura não foi encontrada nenhuma citação que pudesse ser comparada com os dados desta pesquisa.

Nas disfagias orofaringeanas, as complicações mais difíceis de gerenciamento clínico são as afecções pulmona-

Tabela2. Ocorrênciadeaspiraçãoem cada consistência testada.

\begin{tabular}{ccc}
\hline $\begin{array}{l}\text { Consitências aspiradas durante a realização do exame } \\
\begin{array}{c}\text { Pudim/Muido } \\
\text { simultâneas }\end{array}\end{array}$ & $\begin{array}{c}\text { Lelquido } \\
\text { Líquido } \\
\text { simultâneas }\end{array}$ \\
\hline $2(28,5)$ & $3(42,8)$ & $2(28,5)$ \\
\hline
\end{tabular}

Fonte:Aautora

Nota: Número de pacientes(\%). res causadas pela aspiração. Assim, a detectabilidade e caracterização dessa aspiração, que ocorre na fase faríngea, são primordiais para o prognóstico e reabilitação. Pode-se inferir a aspiração pela avaliação clínica, mas sua comprovação objetiva deve ser realizada com a videofluoroscopia (1).

A videofluoroscopia da deglutição é considerado o melhor método instrumental de avaliação da deglutição em crianças, pois avalia desde o início da fase preparatória oral até a conclusão da fase esofágica (2).

A Tabela 1 mostra que das 16 crianças avaliadas, 7 (43,75\%) apresentaram aspiração traqueal, o que segundo a literatura seria um potencializador para a ocorrência de pneumonia aspirativa, pois de acordo com o AHCPR, órgão governamental americano, pacientes disfágicos que aspiram tem risco 50\% maior de desenvolver pneumonia aspirativa, comparado com os pacientes que não aspiram (5).

Dentre a população pesquisa $5(31,25 \%)$ crianças foram classificadas com disfagia grave e alguns trabalhos apontam que crianças com disfagia grave possuem alto risco de complicações durante o exame (3).

No presente trabalho quando se observa a Tabela 2, vemos que a consistência mais aspirada na realização do exame foi a líquida, e a de menor ocorrência de aspiração foi o pudim, indicando que o líquido tem maior incidência de aspiração o que de acordo com a literatura seria outro complicador, pois o risco de o paciente apresentar pneumonia aspirativa aumenta quando a consistência aspirada é a liquida. Em um estudo, no qual realizaram uma retrospectiva dos exames de videofluoroscopia da deglutição realizados por 150 crianças com disfunção da deglutição, houve maior números de casos com pneumonia as crianças que aspiraram consistências mais liquidas, diferente dos resultados das que aspiraram no líquido

Tabela 3. Ocorrência de sintomas respiratórios pós estudo da deglutição em pacientes com disfagia leve, moderada e grave.

\begin{tabular}{|c|c|c|c|c|c|c|}
\hline \multirow{3}{*}{$\begin{array}{l}\text { Sinaise } \\
\text { sintomas } \\
\text { após exame }\end{array}$} & \multicolumn{6}{|c|}{ Grau de disfagia pela classificação de OTT (1996) } \\
\hline & \multicolumn{2}{|c|}{ Leve } & \multicolumn{2}{|c|}{ Moderada } & \multicolumn{2}{|c|}{ Grave } \\
\hline & A & $P$ & A & $P$ & A & $P$ \\
\hline Tosse com catarro & $4(100 \%)$ & & & I (50\%) & & I (20\%) \\
\hline Febre & $4(100 \%)$ & & | (50\%) & & $4(80 \%)$ & \\
\hline Dificuldade respiratória & $4(100 \%)$ & & | (50\%) & & $4(80 \%)$ & \\
\hline $\begin{array}{l}\text { Diminuição na ingestão } \\
\text { dealimento }\end{array}$ & $4(100 \%)$ & & & | (50\%) & & | (20\%) \\
\hline
\end{tabular}

Fonte:Aautora

Nota: $A=$ Ausente; $P=$ Presente; Número de pacientes(\%). 
espesso e no purê, que foram menores os casos de pneumonia (13).

Das $7(43,75 \%)$ crianças que aspiraram durante a realização do estudo da deglutição, somente 2 (12.50\%) crianças a presentaram alguma queixa de sintoma respiratório após 1 semana, porém uma criança já apresentava o mesmo sintoma antes da avaliação, e a outra criança apesar de ter aspirado todas as consistências e ter sido classificada com disfagia grave, não teve diagnóstico positivo de pneumonia em exame físico, contrariando o que dizem literaturas já citados anteriormente.

Esses resultados também mostram o contrário do que afirmam outros autores (14), os quais dizem que uma das complicações em se realizar o estudo da deglutição por videofluoroscopia é que o paciente com o distúrbio poder estar exposto ao risco de aspiração do contraste, e na presente pesquisa é possível observar um número pequeno de complicações pulmonares após o exame (Tabela 3), e sem diagnóstico para pneumonia.

Com isso podemos responder a dois grandes questionamentos, que impulsionaram esta pesquisa:

a) $\mathrm{O}$ exame da deglutição por videofluoroscopia potencializa o risco de pneumonia?

b) O risco beneficio justifica a realização do procedimento?

Vale esclarecer aqui que, durante a avaliação da videofluoroscopia, observa-se apenas uma parte fracionada da deglutição, não podendo assim inferir efetivamente a ocorrência ou não da aspiração durante o processo de deglutição, podendo somente inferir se aspirou ou não naquela fração de tempo. Porém, ainda assim continua sendo o melhor método para avaliar a deglutição em crianças, pois a presente pesquisa mostrou indícios de seguridade do procedimento, mesmo em crianças com disfagia grave, e além do mais, é importante também salientar que a videofluoroscopia pode ter sobre a avaliação clínica não somente caráter complementar, mas também de auxílio na determinação de condutas terapêuticas de forma mais objetiva (1).

\section{CONCLUSÃO}

A videofluoroscopia da deglutição vem sendo o método instrumental mais utilizado concomitantemente à avaliação clinica da deglutição.Porém vale ressaltar a importância de uma equipe multidisciplinar especializada para a realização do exame.

No presente estudo pode-se observar indícios de seguridade do procedimento, mas para que se possa vir a afirmar esse questionamento com maior exatidão, faz-se necessário um número maior de população pesquisada, ficando essa entre outras como sugestão para continuidade de pesquisa na área, bem como correlacionar os dados do exame com as doenças de base.

Na população estudada, não houve ocorrência de pneumonia aspirativa após a realizaçãodoestudoda deglutição por videofluoroscopia, apesar da ocorrência de aspiração durante o exame em cerca de 50\% dos casos (7/16).

\section{AGRADECIMENTOS}

Os autores agradecem ao Dr. Elmar Allen Fugmann por ceder o setor para a pesquisa, e aos profissionais fonoaudiólogos e radiologistas do mesmo setor.

\section{REFERÊNCIAS BILIOGRÁFICAS}

1. Furkim AM, Behlau MS, Weckx LLM. Avaliação clínica e videofluoroscópica da deglutição em crianças com paralisia cerebral tetraparética espástica. Arq Neuropsiquiatr. 2003, 61:611-616.

2. Singh V, et al. Investigation of aspiration: milk nasendoscopy versus videofuoroscopy. Eur Arch Otorhinolaryngol. 2009, 266:543-545.

3. Levy DS, Cristovão PW, Gabbi S. Protocolo do estudo da deglutição por videofluoroscopia. In: Jacobi SJ, Levy DS, Silva LMC. Disfagia: Avaliação e tratamento. RJ: Editora Revinter, 2004. p137.

4. Ramsey D, Smithard D, Kalra L. Silent Aspiration: What Do We Know? Dysphagia. 2005, 20:218-225.

5. Gomes GF. Identificação de fatores preditivos de pneumonia aspirativa em pacientes hospitalizados com doença cerebrovascular complicada por disfagia orofaríngea, Curitiba. 2001 (Tese de Doutorado - Universidade Federal doParaná).

6. Marik PE. Aspiration pneumonitis and aspiration pneumonia. New England J Med. 2001, 344(9):665-671.

7. Mello-Filho M, Silva DD, Issa RE. Videofluoroscopia da deglutição em crianças. In: Jotz GP, Angelis EC, Barros APB. Tratado de deglutição e disfagia - No adulto e na criança. RJ: Editora Revinter, 2009. p.94-96.

8. Maempel IZ, Chapple CL, Leslie P. Radiation Dose in Videofluoroscopic Swallow Studies. Dysphagia. 2007, 22:1315. 
9. Corbin-Lewis K. Liss JM. Sciortino KL. Bases fisiológicas da disfagia neurogênica e estratégias de tratamento. In: Anatomia clínica e fisiologia do mecanismo da deglutição. SP: Cengage Learning, 2009. p.161.

10. Crary MA. A direct intervention program for chronic neurogenic dysphagia secondary to brainstem stroke. Dysphagia, New York, 1995: 6-18.

11. ADA. National Dysphagia Diet: Standardization for Optimal Care. American Dietetic Association. 2002; V-1.
12. Ott DJ, Hodge RG, Pikna LA, Chen MYM and Gelfand DW. Modified barium swallow: clínical and radiographic correlation and relation to feeding recommendations. Dysphagia. 1996, 11:187-190.

13. WEIR, K. et al. Oropharyngeal Aspiration and Pneumonia in Children. Pediatric Pulmonology. 2007, 42:1024-1031.

14. Eckley CA, Fernandes AM. Método de avaliação Otorrinolaringológica da deglutição. ACTA ORL/Técnicas em Otorrinolaringologia. 2005, 23:12-16.

\title{
OCORRÊNCIA DE PNEUMONIA ASPIRATIVA APÓS REALIZAÇÃO DO ESTUDO DA DEGLUTIÇÃO POR VIDEOFLUOROSCOPIA EM CRIANÇAS DE ALTO RISCO
}

\section{Occurrence of Aspiration pneumonia after the performance of the swallow study by videofluoroscopy in infants of high risk}

\author{
Hellen Nataly Correia Lagos, Rosane Sampaio Santos, Adriane Celli, \\ Edna Marcia Silva Abdulmassih, Carlos Alberto do Amaral Medeiros \\ Universidade Tuiuti do Paraná \\ Curitiba - Paraná - Brasil
}

Artigo original

\begin{abstract}
Introdução: a literatura relata que quando se trata de avaliação instrumental da deglutição em crianças, sem dúvida alguma, a videofluoroscopia da deglutição oferece grandes vantagens sobre o estudo endoscópico, além de ser tecnicamente mais factível, reproduz praticamente a situação real da deglutição, porém não se encontram relatos na literatura dos riscos de uma aspiração durante o exame potencializar a ocorrência de pneumonia aspirativa (MELLO-FILHO, et al, 2009). Objetivo: verificar a ocorrência de pneumonia aspirativa após a realização do exame de videofluoroscopia, em crianças de alto risco. Método: participaram do estudo 16 crianças, com faixa etária entre 6 meses e 10 anos, encaminhadas com queixa de disfagia pelo setor de pediatria, para a realização do exame de videofluoroscopia da deglutição. Antes da realização do exame as crianças foram submetidas a radiografia de tórax para excluir possibilidade de pneumonia prévia. Foi realizado retorno após uma semana para avaliação clínica médica e fonoaudiológica e em suspeita de pneumonia encaminhados a radiografia de tórax. Este estudo foi aprovado pelo Comitê de Ética em Pesquisa, sob o n . 000025/2009, da Universidade Tuiuti do Paraná. Para classificação do grau de disfagia, foi utilizada a Classificação da Gravidade da Disfagia à Videofluoroscopia de OTT et al. (1996), na qual classifica-se de Normal, Disfagia Leve, Moderada e Grave. Resultados: dos sintomas respiratórios antes do exame, 2 crianças apresentaram tosse com catarro e dificuldade respiratória, 1 apresentou febre e tosse seca e 1 apresentou somente tosse com catarro. E quanto aos pacientes que apresentaram sintomas respiratórios somente depois da realização do exame, somente um paciente havia aspirado material do exame, porém sem diagnóstico positivo de pneumonia. Com relação ao grau de disfagia, houveram 5 crianças normais, 4 apresentaram disfagia leve, 2 apresentaram moderada e 5 apresentaram grave. Durante a realização do exame 7 pacientes apresentaram aspiração na consistência líquida, sendo que 2 desses pacientes aspiraram também na consistência sólida, 4 também aspiraram nas consistências pastosa e pastosa fina e os 5 restantes não aspiraram. Conclusão: no presente trabalho pode-se concluir que, dentre a população pesquisada não houve a ocorrência de pneumonia aspirativa após o exame.
\end{abstract}

Descritores: transtorno de deglutição; fluoroscopia; pneumonia aspirativa 
Anexo II.

TERMO DE CONSENTIMENTO LIVRE E ESCLARECIDO

Você esta sendo convidado (a) para participar de uma pesquisa. As informações existentes neste documento são para que você entenda perfeitamente os objetivos da pesquisa, e saiba que esta participação é espontânea. Se durante a leitura deste documento houver alguma dúvida você, deve fazer perguntas para que possa entender perfeitamente do que se trata. Após ser esclarecido (a) sobre as informações a seguir, no caso de aceitar fazer parte do estudo, assine ao final este documento, que está em duas vias, sendo uma via sua e a outra do pesquisador responsável.

Informações sobre a Pesquisa:

Título do Projeto de Pesquisa: "Ocorrência de pneumonia aspirativa após realização do estudo da deglutição por videofluoroscopia em crianças de alto risco"

Pesquisador Responsável: Rosane Sampaio Santos

Telefone para Contato: (41) 3331- 7807

\section{INTRODUÇ̃̃O}

A disfagia funcional aspirativa é o distúrbio mais temido, provocado pela aspiração, que é passagem de material abaixo das pregas vocais (BOTELHO, M.I.M.R. SILVA, A.A.,2003), e quando isso acontece pode ocorrer além de outros problemas a pneumonia aspirativa

A pneumonia aspirativa desenvolve-se após inalação de material orofaríngeo contaminado. Apesar de este mecanismo ser comum à maioria das pneumonias a expressão pneumonia aspirativa refere-se ao desenvolvimento de infiltrado pulmonar radiograficamente evidente em pacientes com risco aumentado de aspiração orofaríngea (MARIK, P.E)

O procedimento mais usado para investigação da disfagia é o estudo dinâmico da deglutição (LEVY, D. S. CRISTOVÃO, P. W. GABBI，S，2004).

\section{FINALIDADE DA PESQUISA}

Este trabalho tem como objetivo verificar a ocorrência de pneumonia aspirativa após a realização do exame de videofluoroscopia, em crianças por meio de acompanhamento após a realização do mesmo

\section{PROCEDIMENTO}

O paciente é encaminhado para o setor de endoscopia Per Oral do Hospital de Clínicas - UFPR, para a realização do exame da deglutição por videofluoroscopia, antes da realização do exame serão pegos dados do paciente, através de entrevista aos pais ou responsável, e por informações coletadas do prontuário

O paciente fará então o exame, e os dados serão coletados por meio de um protocolo.

Em uma semana o paciente deve retornar ao mesmo setor para acompanhamento fonoaudiológico e pediátrico, e será avaliado com relação aos sinais de disfagia, histórico de aspiração e condições clínicas, e quando necessário será encaminhado à radiografia de tórax, para confirmar a pneumonia por aspiração

\section{RISCOS E BENEFÍCIOS}

O exame é rápido e indolor. Os achados desta pesquisa poderão elucidar questões sobre os riscos de pneumonia após a realização do exame, tendo em vista que esse exame tem grande importância diagnóstica para avaliação de disfagia

\section{DESCONFORTO}

O exame da deglutição por videofluoroscopia tem como inconveniente o sabor das consistências que serão apresentadas, compostas de água e sulfato de bário.

CUSTOS

Você não terá nenhum gasto com a pesquisa, porque ela será custeada pela própria pesquisadora

\section{PARTICIPACÃ̃O}

Caso você queira desistir da participação na pesquisa, poderá fazê-lo em qualquer tempo e no momento em que desejar.

Todos os participantes da pesquisa serão avaliados pela pesquisadora: Hellen Nataly Correia Lagos graduanda do curso de fonoaudiologia a qual estará sob orientação da fonoaudióloga Rosane Sampaio Santos

Durante o decorrer da pesquisa, caso você venha a ter alguma dúvida ou precise de alguma orientação a mais, use o telefone acima.

\section{PRIVACIDADE E CONFIDENCIALIDADE}

Você tem o compromisso do pesquisador de que a sua imagem e identidade serão mantidas em absoluto sigilo

\section{RESPONSABILIDADE}

Caso ocorra algum tipo de dano no decorrer da pesquisa, as pesquisadoras Rosane Sampaio Santos e Hellen Nataly Correia Lagos se responsabilizam pelos eventuais ressarcimentos.

No caso de novas informações no decorrer da pesquisa, estas serão submetidas à avaliação da Comissão de Ética para um novo parecer.

\section{DECLARAÇÃo DE CONSENTIMENTO}

$\mathrm{Eu}$

portador (a) do RG: abaixo assinado, concordo que meu filho (a)

participe do estudo acima descrito como sujeito.

Fui devidamente informado (a) e esclarecido (a) pelas pesquisadoras Hellen Nataly Correia Lagos e Rosane Sampaio Santos sobre a pesquisa e os procedimentos nela envolvidos.

Foi-me garantido que posso retirar meu consentimento a qualquer momento, sem que isto leve a qualquer penalidade.

Curitiba,

Assinatura do Responsável

Assinatura da Pesquisadora Responsável 